\title{
Antibody-drug Conjugate ADC XMT-1536
}

National Cancer Institute

\section{Source}

National Cancer Institute. Antibody-drug Conjugate ADC XMT-1536. NCI Thesaurus.

Code C147577.

A proprietary antibody-drug conjug ate (ADC) composed of XMT-1535, a proprietary, humanized monoclonal antibody against human sodium-dependent phosphate transport protein 2B (SLC34A2; NaPi2b), site-specifically linked, via a protease cleavable linker, to the proprietary cytotoxic aurastatin derivative auristatin F-HPA (AF-HPA; auristatin Fhydroxypropylamide), with potential antineoplastic activity. XMT-1536 is produced via the proprietary dolaflexin ADC conjug ation platform, which promotes the conjugation of between 10 and 15 AF-HPA payload molecules to each XMT-1535 antibody. Upon administration of XMT-1536, the antibody moiety targets and binds to NaPi2 b expressed on tumor cells. Upon binding, internalization by endosomes/lysosomes, and enzymatic cleavage, the AF-HPA binds to tubulin and inhibits microtubule polymerization, which results in G2/M phase arrest and apoptosis of NaPi2b-expressing tumor cells. NaPi2b, a tumor-associated antigen (TAA), is overexpressed on a variety of cancer cells and plays a key role in the transport of inorganic phosphate ( $\mathrm{Pi}$ ) and the maintenance of phosphate homeostasis. 